\title{
Electric field control of interaction between magnons and quantum spin defects
}

\author{
Abhishek B. Solanki, ${ }^{1,2,}$ ' Simeon I. Bogdanov $\odot,{ }^{1,2,3,4}$ Mohammad M. Rahman, ${ }^{1}$ Avinash Rustagi, ${ }^{1}$ Neil R. Dilley, ${ }^{2}$ \\ Tingting Shen, ${ }^{5,2}$ Wenqi Tong $\odot,{ }^{1}$ Punyashloka Debashis $\odot,{ }^{1,2,6}$ Zhihong Chen $\odot,{ }^{1,2}$ Joerg Appenzeller, ${ }^{1,2}$ \\ Yong P. Chen, ${ }^{5,1,2,7,8,9,10}$ Vladimir M. Shalaev, ${ }^{1,2,7,10}$ and Pramey Upadhyaya ${ }^{1,2,7,10, \dagger}$ \\ ${ }^{1}$ School of Electrical and Computer Engineering, Purdue University, West Lafayette, Indiana 47906, USA \\ ${ }^{2}$ Birck Nanotechnology Center, Purdue University, West Lafayette, Indiana 47906, USA \\ ${ }^{3}$ Department of Electrical and Computer Engineering, University of Illinois at Urbana-Champaign, Urbana, Illinois 60801, USA \\ ${ }^{4}$ Nick Holonyak, Jr. Micro and Nanotechnology Laboratory, University of Illinois at Urbana-Champaign, Urbana, Illinois 61801, USA \\ ${ }^{5}$ Department of Physics and Astronomy, Purdue University, West Lafayette, Indiana 47906, USA \\ ${ }^{6}$ Components Research, Intel Corporation, Hillsboro, Oregon 97124, USA \\ ${ }^{7}$ Purdue Quantum Science and Engineering Institute (PQSEI), Purdue University, West Lafayette, Indiana 47906, USA \\ ${ }^{8}$ Institute of Physics and Astronomy and Villum Center for Hybrid Quantum Materials and Devices, Aarhus University, \\ 8000 Aarhus-C, Denmark \\ ${ }^{9}$ WPI-AIMR International Research Center for Materials Sciences, Tohoku University, Sendai 980-8577, Japan \\ ${ }^{10}$ The Quantum Science Center (QSC), a National Quantum Information Science Research Center of the U.S. Department of Energy (DOE), \\ Oak Ridge National Laboratory, Oak Ridge, Tennessee 37831, USA
}

(Received 24 May 2021; revised 28 December 2021; accepted 26 January 2022; published 23 February 2022)

\begin{abstract}
Hybrid systems coupling quantum spin defects (QSD) and magnons can enable unique spintronic device functionalities and probes for magnetism. Here, we add electric field control of magnon-QSD coupling to such systems by integrating ferromagnet-ferroelectric composite multiferroic with nitrogen-vacancy (NV) center spins. Combining quantum relaxometry with ferromagnetic resonance measurements and analytical modeling, we reveal that the observed electric-field tuning is consistent with the ferroelectric polarization control of the magnon-generated fields at the NV. Exploiting this mechanism, we also propose magnon-based hybrid electric field sensors which provide the possibility of improving dc electric field sensitivity of single-spin sensors.
\end{abstract}

DOI: 10.1103/PhysRevResearch.4.L012025

Introduction. Optically active quantum spin defects (QSDs), i.e., microscopic spin impurities in insulating hosts, coupled with magnons, i.e., the elementary collective excitations of macroscopically ordered magnetic systems, have recently emerged as a promising platform [1-20] to explore the paradigm of hybrid quantum systems [21-24]. The motivation for creating magnon-QSD systems is twofold. First, magnons resonantly enhance microwave fields up to nanoscale [1], feature long-distance nonreciprocal transport [25], and exhibit mode confinement at reconfigurable nanoscale magnetic textures [26-28]-properties that have given birth to the field of magnonics [29]. Therefore, magnons provide promising control fields for solving the challenge of on-chip coherent driving $[1,2,13,30]$ and communication between QSD qubits [3,4,31]. Second, application of well-established quantum defect magnetometry techniques to magnon-generated fields enables previously unavailable

\footnotetext{
*solanka@purdue.edu

†prameyup@purdue.edu

Published by the American Physical Society under the terms of the Creative Commons Attribution 4.0 International license. Further distribution of this work must maintain attribution to the author(s) and the published article's title, journal citation, and DOI.
}

noninvasive and nanoscale probes for a broad range of magnetic phenomena [1-20].

Adding electric field control to QSD-magnon coupling would expand the range of phenomena and device functionalities that can be enabled by magnon-QSD hybrid systems. For example, the dependence of magnon-spin coupling on an applied electric field, when combined with the magnetometry of magnon-generated fields, provides a scheme for sensing electric phenomena via QSDs. Apart from extending the reach of QSD-based nanoscale sensors [32] to probe magnetoelectric materials, the attractive feature offered by this approach includes leveraging magnetic resonance enhancement [5] and the stronger magnetic field susceptibility of QSD ground state [33] to enhance the electric field sensitivity. On another front, electric fields, as opposed to magnetic fields and currents, can be confined to the scale of interqubit separation with minimal joule heating $[13,34,35]$. Electric field tuning of QSD-magnon interaction could thus enable a scalable network of QSDbased circuits, where the desired spins are driven and/or entangled with their neighbors via locally tunable magnon modes.

In this Letter, we propose and study a hybrid system that combines QSDs with a ferromagnetic-ferroelectric composite multiferroic [36-39], the main results of which can be summarized as follows. First, we experimentally demonstrate electric field control of interaction between magnons and QSDs in this hybrid system. Second, we show that the observed control 


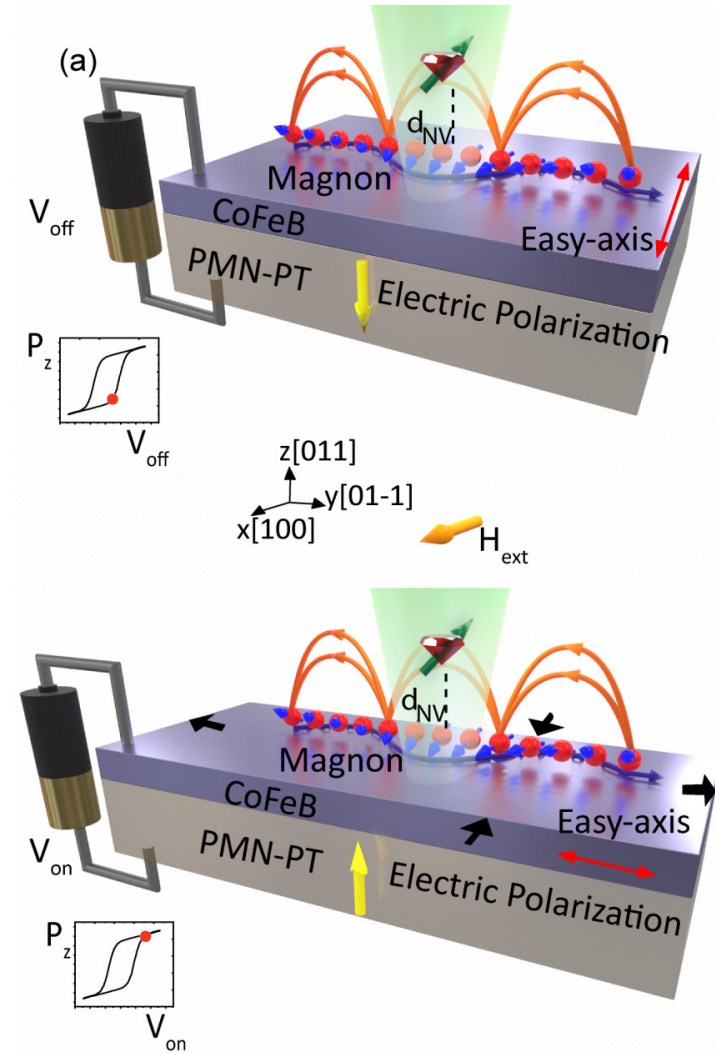

(b)
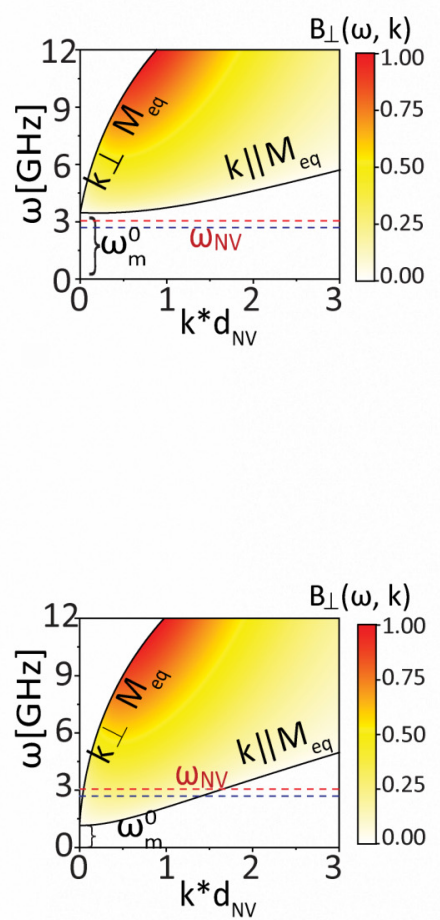

(c)
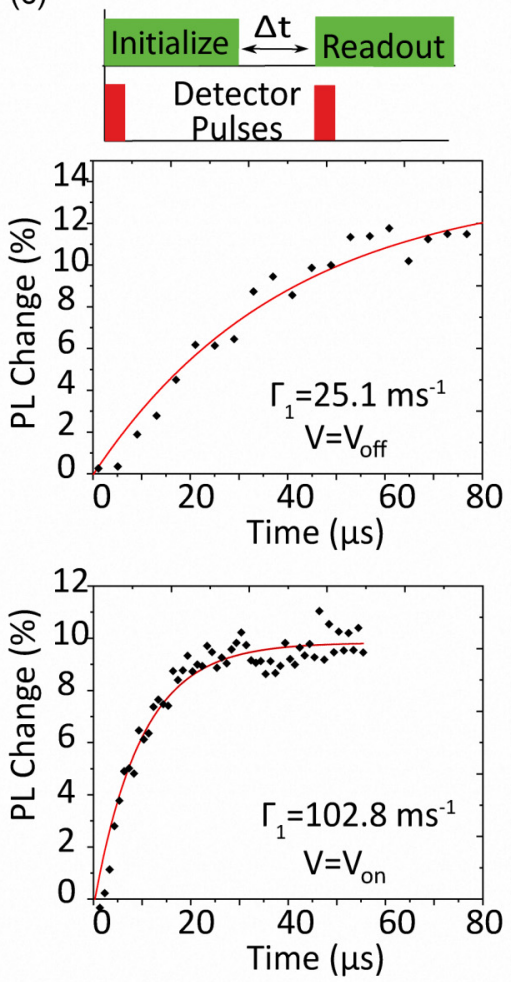

FIG. 1. (a) Schematic illustration of the QSD-magnon hybrid system. An external voltage $V$ controls the electrical polarization of PMN-PT (as shown in schematic P- $V$ loops) resulting in lattice strain and reorientation of the magnetic anisotropy field in CoFeB (easy axis-red double arrows) from $x$ axis for $V=V_{\text {off }}$ to $y$ axis for $V=V_{\text {on }}$. (b) Maps of normalized $B_{\perp}(k)$ as a function of $\omega-k$ for both $V_{\text {off }}$ and $V_{\text {on }}$. The black lines enveloping the color map are the calculated magnon dispersion lines for bulk modes $(k \| M)$ and surface modes $(k \perp M)$. The dashed colored lines represent the NV ESR lines $\omega_{\mathrm{NV}}$. (c) Change in relaxation rates of the NV ensembles enabled by electrical tuning of QSD-magnon coupling. The schematic diagram represents the pulse sequence of the measurement scheme.

is consistent with the mechanism of electric field tuning of magnon-generated fields at the QSD, which in turn results from the coupled ferroelectric, elastic, and magnetic orders in the composite multiferroic. Last, we show theoretically that in nanomagnet-ferroelectric-QSD hybrid systems this mechanism provides a possibility to achieve enhancement in single-spin dc electric field sensitivity over schemes using direct coupling of electric fields to QSDs [40].

Central scheme. We disperse nanodiamonds with nitrogen vacancy (NV) center [41] ensembles, which act as QSDs, onto a ferromagnetic (FM)/ferroelectric (FE) heterostructure, which is formed by combining $(20 \mathrm{~nm}) \mathrm{CoFeB}$ and (300 $\mu \mathrm{m}, 011$-cut) $\mathrm{Pb}\left(\mathrm{Mg}_{1 / 3} \mathrm{Nb}_{2 / 3}\right) \mathrm{O}_{3}-\mathrm{PbTiO}_{3}$ (PMNPT) films [Fig. 1(a)]. By integrating the magnetoelastic and piezoelectric properties of FM and FE films, such FM/FE heterostructures behave as a multiferroic (referred to as a composite multiferroic $[38,39]$ ). The magnons in $\mathrm{CoFeB}$ couple with QSDs by producing an oscillating dipole magnetic field at the NV, whose magnitude and frequency $\left(\omega_{m}\right)$ depend on their wave vector $(k)$ [6]. The central idea we exploit here is that by controlling the ferroelectric polarization component $P_{z}$ along the [011] axis in PMN-PT, magnon bands in CoFeB film can be moved with respect to the NV ESR transitions, thereby enabling the sought electric-field control.

As an example, we show in Fig. 1(b) the particular case of how the magnon bands respond to the flipping of $P_{z}$ from
$-z\left(V=V_{\text {off }}\right)$ to $+z\left(V=V_{\text {on }}\right)$ direction in the presence of a fixed external magnetic field $\left(H_{\text {ext }}\right)$ along the [100] $x$ axis. The (011)-cut PMN-PT features piezoelectric coefficients $d_{31}$ and $d_{32}$ of opposite signs. Consequently, to accommodate the increasing $P_{z}$, PMN-PT expands along the $\left[\begin{array}{lll}0 & 1 & -1\end{array}\right] y$ axis and shrinks along the [100] $x$ axis. The transfer of this anisotropic strain to $\mathrm{CoFeB}$, when combined with the magnetoelastic interaction, imprints a dynamically tunable magnetic anisotropy with the easy-axis reorienting from $x$ to $y$ for $P_{z}$ changing from $-z$ to $+z$ [38,39]. Crucially, the magnon band gap $\omega_{m}^{0} \equiv \omega_{m}(k=0)$ and the frequency range spanned by the magnon bands depend on the magnetic anisotropy [42]. When $H_{\text {ext }}$ is oriented along the easy axis $\omega_{m}^{0}$ is maximized, while the orthogonal orientation minimizes $\omega_{m}^{0}$. Consequently, as the voltage is increased from $V_{\text {off }}$ to $V_{\text {on }}$ the magnon bands are pulled to lower frequencies and brought into resonance with the NV ESR transitions [see Fig. 1(b)].

To demonstrate the above mechanism we perform NVrelaxometry [41] measurements in the presence of thermal magnons. Here, the coupling to the thermal magnons acts as a source of magnetic noise for the NV spins. This results in an increased NV spin relaxation rate $\left(\Gamma_{1}\right)$ (from its intrinsic value of $\Gamma_{0}=3 \mathrm{~ms}^{-1}$ ), which thus provides a measure of the magnon-QSD coupling. In the remainder of this Letter, we demonstrate this electrical tuning of spin-magnon coupling [see Fig. 1(c)], along with highlighting its implication 
(a)

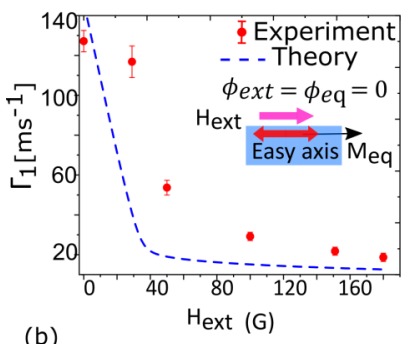

(b)

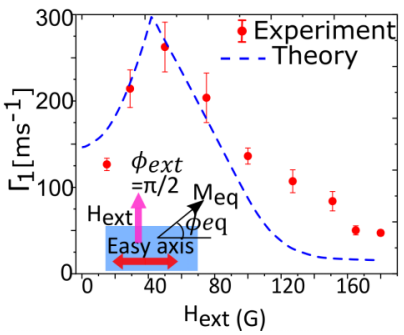

(c)

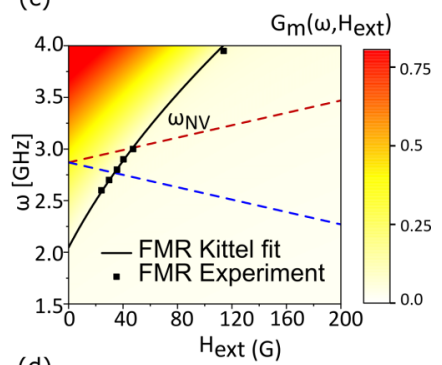

(d)

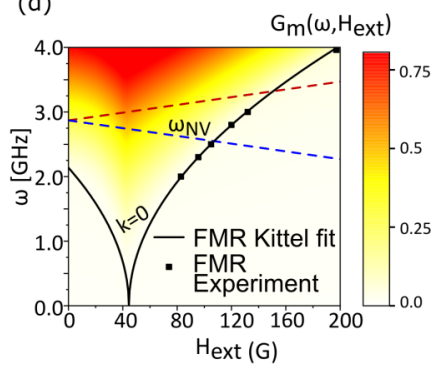

FIG. 2. (a), (b) Measured relaxation rate $\Gamma_{1}$ as a function of external magnetic field $H_{\text {ext }}$ applied parallel ( $x$ axis) and orthogonal ( $y$ axis) to the magnetic anisotropy field (easy axis), respectively. The dashed lines represent theoretical fits of relaxation rates $\Gamma_{1}$. The inset shows the schematic variation of $H_{\mathrm{ext}}$ with respect to the easy axis and the direction of equilibrium magnetization. (c), (d) Ferromagnetic resonance (FMR) frequency $\left(\omega_{m}^{0}\right)$ as a function of external magnetic field $H_{\text {ext }}$ fitted with the Kittel formula for $20 \mathrm{~nm}$ CoFeB film (solid lines) for $H_{\text {ext }}$ parallel ( $x$ axis) and orthogonal ( $y$ axis) to the anisotropy field, respectively. The dashed colored lines represent maximum spread of the NV ESR lines $\omega_{\mathrm{NV}}$. The color map represents the calculated values of the magnetic noise spectral density $G_{m}\left(\omega, H_{\text {ext }}\right)$ for an effective NV height $d_{\mathrm{NV}}=77 \mathrm{~nm}$.

for improving QSD-based sensors of electric fields and phenomena.

Anisotropic QSD-magnon coupling. We begin by understanding the role of a static magnetic anisotropy field on $\mathrm{NV}$-spin magnon coupling. To this end, we measure the relaxation rate $\Gamma_{1}$ for $H_{\text {ext }}$ applied along the [100] $x$ axis and the $[01-1] y$ axis of the (011-cut) PMN-PT [Figs. 2(a) and 2(b)]. For the field applied along the $x$ axis, $\Gamma_{1}$ decreases monotonically reaching the minimum value of $18.7 \pm 2 \mathrm{~ms}^{-1}$ for $H_{\mathrm{ext}}=180 \mathrm{G}$. On the other hand, for the field applied along the $y$ axis, $\Gamma_{1}$ increases first reaching a maximum value of $262.4 \pm 29 \mathrm{~ms}^{-1}$ at $H_{\mathrm{ext}}=50 \mathrm{G}$ and then decreases.

The relaxation of NV spins coupled to magnons is governed by the spectral density of magnon-generated transverse field fluctuations evaluated at the NV ESR transitions [6], which is given by

$$
G_{m}(\omega)=\int B_{\perp}^{2}(k) D\left[\omega, \omega_{m}(k)\right] n\left[\omega_{m}(k)\right] A d k /(2 \pi)^{2} .
$$

Here, $B_{\perp}$ is the magnitude of the transverse magnetic field at the NV due to a magnon occupying the mode with frequency $\omega_{m}$ and wave vector $k$ [whose relative strength is shown in Fig. 1(b)], $D=\alpha \omega_{m} / \pi\left[\left(\omega-\omega_{m}\right)^{2}+\alpha^{2} \omega_{m}^{2}\right]$ is the magnon spectral density with $\alpha$ being the Gilbert damping parameter, and $n A d k / 2 \pi^{2}$ counts the total number of thermal magnons occupying the states in the neighborhood of $k$, where $A$ is the

area of the film and $n=k_{B} T / \hbar \omega_{m}$ is the Rayleigh-Jeans distribution function with $k_{B}$ and $T$ being the Boltzmann constant and temperature, respectively. External magnetic field tunes the spectral density of magnetic noise resonant with the NV ESR transitions by controlling the magnon spectrum.

The magnon spectrum in thin films is described by the dipole-exchange spin waves [43]: $\omega_{m}(k)=\omega_{m}^{0}+f(k)$. Here, $\omega_{m}^{0}$ (Kittel mode) is the band gap at $k=0$ and $f(k)$ (see Supplemental Materials S4) describes the nondegenerate branches of dispersion [cf. magnon bands in Fig. 1(b)]. To track the location of magnon bands, which is needed for the calculation of magnon-generated noise spectrum as per Eq. (1), we present the results of ferromagnetic resonance (FMR) experiments on our multiferroic films in Figs. 2(c) and 2(d) (see Supplemental Materials S1 for details of FMR experiments). FMR directly measure $\omega_{m}^{0}$ as a function of $H_{\text {ext }}$ along the $x$ and $y$ axis. Consistent with our relaxometry measurements, $\omega_{m}^{0}$ also depends on the orientation of $H_{\text {ext }}$, lying at a higher frequency for $H_{\text {ext }} \| x$ when compared with that for $H_{\text {ext }} \| y$.

This anisotropic behavior can be described by considering a uniaxial magnetic anisotropy energy of the form $\mathcal{F}_{a n}=$ $H_{k} M_{s}\left(m_{y}^{2}-m_{x}^{2}\right) / 2[36,38,39]$ in the magnetic film. Here, $M_{s}$ is the saturation magnetization, $m_{x}$ and $m_{y}$ are the $x$ and $y$ components of the unit vector oriented along the magnetization, and $H_{k}$ is the uniaxial anisotropy field. In such films, $\omega_{m}^{0}$ is governed by the Kittel formula [42]:

$$
\omega_{m}^{0}=\gamma \sqrt{H_{1} H_{2}},
$$

with $H_{1}=H_{\text {ext }} \cos \left(\phi_{\text {ext }}-\phi_{e q}\right)+2 H_{k} \cos 2 \phi_{e q}$ and $H_{2}=$ $H_{\text {ext }} \cos \left(\phi_{\text {ext }}-\phi_{e q}\right)+H_{k} \cos 2 \phi_{e q}+4 \pi M_{s}$. Here, $\phi_{\text {ext }}$ and $\phi_{e q}$ are the azimuthal angles of the external magnetic field and the equilibrium magnetization, respectively [see the inset of Figs. 2(a) and 2(b)]. The Kittel formula fits are shown in Figs. 2(c) and 2(d), which gives $H_{k}=20 \mathrm{G}$ with the easy axis oriented along the $x$ axis.

Substituting the magnon spectrum parameters from the FMR measurements in Eq. (1), we plot the normalized fielddependent noise spectral density $G_{m}\left(\omega, H_{\text {ext }}\right)$, along with the maximum spread of $\mathrm{NV}$ ensemble ESR frequencies $\omega_{\max }^{+}=2.87+\gamma H_{\text {ext }} \mathrm{GHz}$ and $\omega_{\text {min }}^{-}=2.87-\gamma H_{\text {ext }} \mathrm{GHz}$, in Figs. 2(c) and 2(d). For $H_{\text {ext }} \| x$, magnons generating higher $B_{\perp}$ are pushed away from the frequency range probed by the $\mathrm{NV}$ ensemble due to the monotonic shift of magnon bands to higher frequency with $H_{\text {ext }}$ [see Fig. 1(b)]. As a result, for $H_{\text {ext }} \| x, G_{m}\left(\omega, H_{\text {ext }}\right)$ within $\omega_{\max }^{+}$and $\omega_{\min }^{-}$decreases monotonically, consistent with the relaxation rate's decrease with the field whereas, for $H_{\text {ext }} \| y$, since $\omega_{m}^{0}$ is first pushed to lower frequencies for $H_{\text {ext }}<50 \mathrm{G}$ before moving to higher frequencies for $H_{\text {ext }}>50 \mathrm{G}$, magnons with stronger $B_{\perp}$ are brought into (out of) resonance with $\mathrm{NV}$ ensemble ESR transitions for $H_{\text {ext }}<50 \mathrm{G}\left(H_{\text {ext }}>50\right) \mathrm{G}$. Consequently, $G_{m}\left(\omega, H_{\text {ext }}\right)$ within $\omega_{\max }^{+}$and $\omega_{\min }^{-}$and the relaxation rates increase (decrease) with $H_{\text {ext }}$ for $H_{\text {ext }}<50 \mathrm{G}\left(H_{\text {ext }}>50\right) \mathrm{G}$. Theoretical best fits of relaxation rate (see Supplemental Materials S4) are also shown in Figs. 2(a) and 2(b) exhibiting good qualitative agreement. Quantitative differences may arise from neglecting finite mode ellipticity for magnons [6,16], two-magnon scattering-induced NV relaxation [11], and/or effect of local inhomogeneities (arising, for example, from local strain) on the magnon spectrum. 


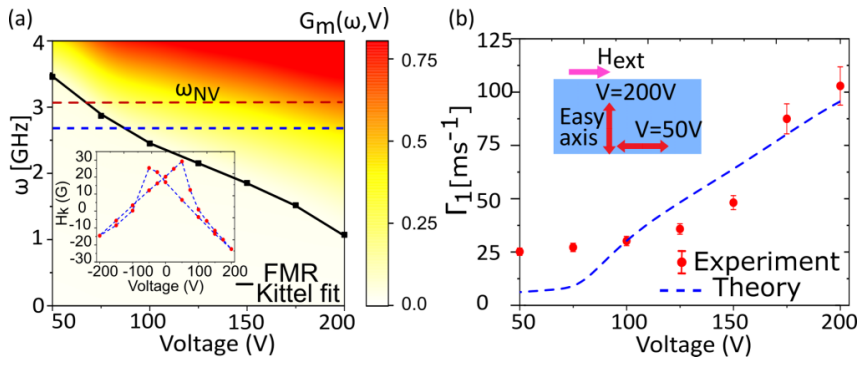

FIG. 3. (a) FMR frequency $\left(\omega_{m}^{0}\right)$ (black line) as a function of applied voltage extracted from the experimental results for a fixed external magnetic field $H_{\mathrm{ext}}=57 \mathrm{G}$ along the $x$ axis. The color map represents the calculated values of the magnetic noise spectral density $G_{m}(\omega, V)$ for an effective NV height $d_{\mathrm{NV}}=77 \mathrm{~nm}$. The dashed colored lines represent maximum spread of the NV ESR lines $\omega_{\mathrm{NV}}$. The inset shows the measured magnetic anisotropy field as a function of applied voltage. We begin by polling the ferromagnet to $-200 \mathrm{~V}$ and then changing the voltage in steps towards $+200 \mathrm{~V}$ and back to $-200 \mathrm{~V}$. (b) Measured relaxation rates $\Gamma_{1}$ as a function of applied voltage for a fixed $H_{\text {ext }}=57 \mathrm{G}$ along the $x$ axis. The inset shows a schematic illustration of the magnetic anisotropy field for the two different voltages for a fixed $H_{\text {ext }}$. The dashed line represents theoretical fit of relaxation rates $\Gamma_{1}$

Electric field control. The multiferroic hybrid offers the attractive property of moving magnon spectrum with electric field by dynamically tuning the magnetic anisotropy. In Fig. 3(a), we show measured $\omega_{m}^{0}(V)$ for $H_{\text {ext }}=57 \mathrm{G}$ along the $x$ axis with $H_{k}(V)$ (see inset) extracted by applying the Kittel formula Eq. (2) on the measured $\omega_{m}^{0}(V)$ (see Supplemental Materials S1). The $H_{k}(V)$ inherits the characteristic butterflyshaped curve of anisotropic strain as a function of applied voltage, which is the hallmark of coupled ferroelectric, elastic, and magnetic order tuning the anisotropy in FM/FE multiferroics $[36,38,39]$. Particularly, as the voltage is increased from $50 \mathrm{~V}$ to $200 \mathrm{~V}, H_{k}$ decreases monotonically from $H_{k}=30 \mathrm{G}$ at $V=50 \mathrm{~V}$ to $H_{k}=-22 \mathrm{G}$ at $V=200 \mathrm{~V}$. Notably, this maximal change in $H_{k}$ corresponds to the flipping of the easy axis from $x$ to $y$ consistent with flipping of $P_{z}$ in PMN-PT (see Supplemental Materials S2). Correspondingly, we observe the largest $\omega_{m}^{0}=3.45 \mathrm{GHz}$ for $V=50 \mathrm{~V}$ when the easy axis is aligned with the direction of $H_{\text {ext }}$ and smallest $\omega_{m}^{0}=1.07 \mathrm{GHz}$ for $V=200 \mathrm{~V}$ when the easy axis is aligned orthogonal to the direction of $H_{\text {ext }}$.

We next obtain the normalized field-dependent magnetic noise spectral density $G_{m}(\omega, V)$ shown in Fig. 3(a) by substituting the electric field-dependent magnon spectrum from FMR experiments into Eq. (1) (see Supplemental Materials S4). We also depict the NV ESR frequencies, which lie within the dashed horizontal lines $\omega_{\max }^{+}\left(H_{\mathrm{ext}}=57\right) \mathrm{GHz}$ and $\omega_{\text {min }}^{-}\left(H_{\text {ext }}=57\right) \mathrm{GHz}$. For $V=50 \mathrm{~V}$, i.e., $P_{z}$ points downward, the $\omega_{m}^{0}=3.45 \mathrm{GHz}$ is the largest for our hybrids and lies above the NV transition frequency [Fig. 3(a)] [as shown in Fig. 1(b), where we referred to $V=50 \mathrm{~V}$ as $\left.V_{\text {off }}\right]$. Consequently, magnons are off resonant with NVs and are expected to couple weakly with them, which is reflected in the low value of calculated $G_{m}(\omega, V)$ within $\omega_{\max }^{+}$and $\omega_{\min }^{-}$band at $V=50 \mathrm{~V}$. As the voltage is increased, the polarization of PMN-PT is reoriented, $H_{k}$ and $\omega_{m}^{0}$ decrease, and magnons with stronger dipole fields are brought into the resonance with the NV ESR transitions (see Fig. 1), which is reflected in $G_{m}(\omega, V)$ increasing monotonically and reaching the maximum value within the frequency range spanned by $\omega_{\max }^{+}$and $\omega_{\text {min }}^{-}$for $V=200 \mathrm{~V}$; we thus refer to $V=200 \mathrm{~V}$ as $V_{\text {on }}$.

The experimentally measured spin relaxation rate indeed increases monotonically as the voltage varies from $V_{\text {off }}$ to $V_{\text {on }}$ [see Fig. 3(b)], showing good agreement with the above qualitative picture. Namely, low relaxation rate of $25.1 \pm 2$ $\mathrm{ms}^{-1}$ is observed for $V=V_{\text {off }}$, which increases monotonically to $102.8 \pm 9 \mathrm{~ms}^{-1}$ at $V=V_{\text {on }}$, a change of $400 \%$. The corresponding optical spin contrast curves are shown in Fig. 1(c). We also plot the results of theoretical $\Gamma_{1}$ fits for the NV ensembles, with the nanodiamond orientation found in the previous section (see Supplemental Materials S4). The theoretical fits agree well with the expected qualitative trend. In addition to the reasons pointed out for Figs. 2(a) and 2(b), inhomogeneous magnon-scattering potentials introduced by ferroelectric domains may contribute to quantitative differences [44]. We also measure relaxation rates of $\mathrm{NV}$ nanodiamonds directly on PMN-PT in the absence of CoFeB films, which shows no observable dependence on the voltage (see Supplemental Materials S3). This further corroborates that the observed tuning is consistent with the electric field modulation of magnon-generated fields.

Discussion. The shift in NV's relaxation rate due to the ferroelectric polarization control of magnon mode frequency can be exploited for sensing E fields ("magnon sensing"). We next compare its expected single-spin dc E-field sensitivity $(S)$ to the scheme based on direct coupling of NV's ESR levels to the dc E field [40] ("direct sensing"). For both schemes, $S \sim$ $1 / C \eta \sqrt{T_{\chi}}$ [45] (see also Supplemental Materials S4). Here, $C$ is the photoluminescence readout efficiency parameter, $\eta$ is the transduction parameter, which for the magnon sensing (direct sensing) is given by $\eta_{m}=\partial \Gamma_{1} / \partial E\left(\eta_{d}=\right.$ Stark shift [40]), and $T_{\chi}$ is the time scale before which unwanted signals dephase or relax the NV spin. For direct sensing, $\eta_{d} \sim 28$ $\mathrm{Hz} /(\mathrm{V} / \mathrm{cm})$ and $T_{\chi} \sim \mathrm{ms}$ is the NV's decoherence time [40]. For our experiments, $\eta_{m} \sim 15 \mathrm{~Hz} /(\mathrm{V} / \mathrm{cm})$ and $T_{\chi}=1 / \Gamma_{1} \sim$ $10 \mu \mathrm{s}$ from the high voltage bias region of Fig. 3(b). This suggests that the sensitivity of the magnon-sensing scheme $S_{m}$ is an order of magnitude poorer than $S_{d}$ for the parameter regime we study here. However, unlike $S_{d}$ (which is limited by the fixed Stark shift), $S_{m}$ can be improved by orders of magnitude by exploiting the magnon modes of nanomagnet (instead of films), as highlighted next.

The transduction parameter for magnon sensing can be written as $\eta_{m}=v \beta$, where $v \equiv \partial \Gamma_{1} / \partial \omega_{m}$ and $\beta \equiv \partial \omega_{m} / \partial E$. In nanoscale magnets, the magnon modes are discretized due to confinement [46]. Consequently, a much smaller shift in the magnon frequency $\left(\sim \alpha \omega_{m}\right)$ would make the magnon mode and the NV ESR off resonant. This enhances $v$ compared to films, where a broad band of magnons must be moved before NV and magnons become off resonant [cf. Fig. 1(b)]. Informed by these heuristics, we calculate $S_{m}$ for an NV center coupled to the Kittel mode of the nanomagnet/PMN-PT hybrid system (see the inset of Fig. 4).

Specifically, we first obtain $v(\omega)$ by differentiating the relaxation rate $\Gamma_{1}=\gamma^{2} / 2 G_{m}^{s}(\omega)$ with respect to frequency. Here, $G_{m}^{s}(\omega) \mid$ the spectral density of the magnetic noise at 


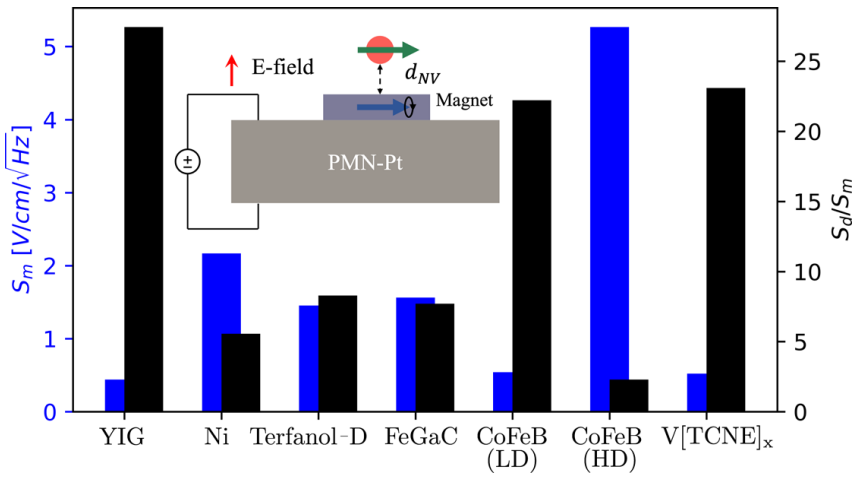

FIG. 4. Estimates of magnon-sensing sensitivity, $S_{m}$ (blue bars), and its comparison to direct-sensing, $S_{d}$ (as quantified by the ratio $S_{d} / S_{m}$; black bars), for various magnetic materials ("HD" and "LD" stand for high damping and low damping, respectively. The material parameters and corresponding references are summarized in the Supplemental Material, Table I). Inset shows the proposed hybrid structure.

the NV due to the nanomagnet- is obtained by taking the single-domain and finite size limit of Eq. (1) (see Supplemental Material section S5, where the calculated $\Gamma_{1}$ is also corroborated against direct numerical simulations). Next, we calculate $\beta$ using the Kittel formula [Eq. (2)] and $\partial H_{K} / \partial E=$ $3 B d_{\text {eff }} / M_{s}$ for the nanomagnet/PMN-PT hybrid system [36]. Here, $d_{\text {eff }}$ is the effective piezoelectric coefficient of PMN-PT and $B=\lambda_{s} Y$ is the magnetoeleastic coefficient of the nanomagnet, with $\lambda_{s}$ and $Y$ being its saturation magnetostriction and Young's modulus, respectively. Substituting these $v$ and $\beta$ into the definition of $\eta_{m}$, and biasing the NV ESR to be resonant with the frequency that maximizes $v$, we obtain $S_{d}$. In Fig. 4 we plot the ratio $S_{d} / S_{m}$ for typical magnetoelastic materials, which shows that for a variety of existing material platforms the calculated $S_{m}<S_{d}$, notably suggesting over an order of magnitude improvement for the recently demonstrated V[TCNE] $]_{x} / \mathrm{PMN}-\mathrm{PT}$ multiferroic [47]. Taking typical values for $C=0.3$ [45,48], we also estimate the order of magnitude of $S_{m}$, which can reach $\sim 1(\mathrm{~V} / \mathrm{cm}) / \sqrt{H} z$.

We remark that, while the quantitative differences between theory and experiments (similar to those observed at the film level) cannot be ruled out, the potential reasons noted for such differences are less likely to apply for the nanomagnet geometry [49]. Thus our calculations suggest that the magnon sensing scheme in nanomagnet-ferroelectric hybrid systems could provide a route to improve dc E-field sensitivities of NV centers. Furthermore, since the magnetoelastic coupling is local [50] and also present for polarization modulation around zero applied bias voltage [38,39], the nanomagnetbased magnon sensing scheme is expected to have nanoscale resolution and find use for applications not having access to large supply voltage (see Supplemental Materials S5 and S6).

Future research extending the present study to the scanning NV geometry [20] could extend the advantages of NV-center probing of condensed matter [32] to multiferroics and a broad range of magnetoelectric phenomena studied in spintronics [34]. Beyond sensing, an array of QSD/nano-FM/FE [51] hybrids could enable scalable QSD-based quantum circuits, where selective driving of QSD qubits by a weak global microwave drive [51] and tunable transfer of information between such qubits can be activated electrically by tuning the magnon frequency of nano-FMs in proximity to the desired qubits.

Acknowledgments. We thank S. W. Kurfman and E. Johnston-Halperin for sharing the details of measurements on the V[TCNE $]_{x} /$ PMN-PT hybrid system. This work was supported in part by NSF Award No. 1838513, NSF Award No. 1944635, NSF Award No. 2015025-ECCS, and the U.S. Department of Energy, Office of Science through the Quantum Science Center (QSC), a National Quantum Information Science Research Center.

A.B.S., S.I.B., and M.M.R. contributed equally to this work.
[1] P. Andrich, F. Charles, X. Liu, H. L. Bretscher, J. R. Berman, F. J. Heremans, P. F. Nealey, and D. D. Awschalom, Long-range spin wave mediated control of defect qubits in nanodiamonds, npj Quantum Inf. 3, 28 (2017).

[2] D. Kikuchi, D. Prananto, K. Hayashi, A. Laraoui, N. Mizuochi, M. Hatano, E. Saitoh, Y. Kim, C. A. Meriles, and T. An, Long-distance excitation of nitrogen-vacancy centers in diamond via surface spin waves, Appl. Phys. Express 10, 103004 (2017).

[3] L. Trifunovic, F. L. Pedrocchi, and D. Loss, Long-Distance Entanglement of Spin Qubits via Ferromagnet, Phys. Rev. X 3, 041023 (2013).

[4] B. Flebus and Y. Tserkovnyak, Entangling distant spin qubits via a magnetic domain wall, Phys. Rev. B 99, 140403(R) (2019).

[5] L. Trifunovic, F. L. Pedrocchi, S. Hoffman, P. Maletinsky, A. Yacoby, and D. Loss, High-efficiency resonant amplification of weak magnetic fields for single spin magnetometry at room temperature, Nat. Nanotechnol. 10, 541 (2015).
[6] T. Van der Sar, F. Casola, R. Walsworth, and A. Yacoby, Nanometre-scale probing of spin waves using single electron spins, Nat. Commun. 6, 7886 (2015).

[7] M. Page, B. A. McCullian, C. Purser, J. G. Schulze, T. M. Nakatani, C. S. Wolfe, J. R. Childress, M. E. McConney, B. M. Howe, P. C. Hammel, and V. P. Bhallamudi, Optically detected ferromagnetic resonance in diverse ferromagnets via nitrogen vacancy centers in diamond, J. Appl. Phys. 126, 124902 (2019).

[8] C. Wolfe, S. Manuilov, C. Purser, R. Teeling-Smith, C. Dubs, P. Hammel, and V. Bhallamudi, Spatially resolved detection of complex ferromagnetic dynamics using optically detected nitrogen-vacancy spins, Appl. Phys. Lett. 108, 232409 (2016).

[9] H. Zhang, M. J. H. Ku, F. Casola, C. H. R. Du, T. van der Sar, M. C. Onbasli, C. A. Ross, Y. Tserkovnyak, A. Yacoby, and R. L. Walsworth, Spin-torque oscillation in a magnetic insulator probed by a single-spin sensor, Phys. Rev. B 102, 024404 (2020). 
[10] C. Du, T. Van der Sar, T. X. Zhou, P. Upadhyaya, F. Casola, H. Zhang, M. C. Onbasli, C. A. Ross, R. L. Walsworth, Y. Tserkovnyak et al., Control and local measurement of the spin chemical potential in a magnetic insulator, Science 357, 195 (2017).

[11] B. McCullian, A. M. Thabt, B. Gray, A. Melendez, M. S. Wolf, V. Safonov, D. Pelekhov, V. Bhallamudi, M. Page, and P. Hammel, Broadband multi-magnon relaxometry using a quantum spin sensor for high frequency ferromagnetic dynamics sensing, Nat. Commun. 11, 5229 (2020).

[12] T. X. Zhou, J. J. Carmiggelt, L. M. Gächter, I. Esterlis, D. Sels, R. J. Stöhr, C. Du, D. Fernandez, J. F. Rodriguez-Nieva, F. Büttner, E. Demler, and A. Yacoby, A magnon scattering platform, Proc. Natl. Acad. Sci. USA 118, e2019473118 (2021).

[13] D. Labanowski, V. Bhallamudi, Q. Guo, C. Purser, B. McCullian, P. Hammel, and S. Salahuddin, Voltage-driven, local, and efficient excitation of nitrogen-vacancy centers in diamond, Sci. Adv. 4, eaat6574 (2018).

[14] B. Flebus and Y. Tserkovnyak, Quantum-Impurity Relaxometry of Magnetization Dynamics, Phys. Rev. Lett. 121, 187204 (2018).

[15] A. Finco, A. Haykal, R. Tanos, F. Fabre, S. Chouaieb, W. Akhtar, I. Robert-Philip, W. Legrand, F. Ajejas, K. Bouzehouane et al., Imaging non-collinear antiferromagnetic textures via single spin relaxometry, Nat. Commun. 12, 767 (2021).

[16] A. Rustagi, I. Bertelli, T. van der Sar, and P. Upadhyaya, Sensing chiral magnetic noise via quantum impurity relaxometry, Phys. Rev. B 102, 220403(R) (2020).

[17] S. Chatterjee, J. F. Rodriguez-Nieva, and E. Demler, Diagnosing phases of magnetic insulators via noise magnetometry with spin qubits, Phys. Rev. B 99, 104425 (2019).

[18] C. Purser, V. Bhallamudi, F. Guo, M. Page, Q. Guo, G. Fuchs, and P. Hammel, Spinwave detection by nitrogen-vacancy centers in diamond as a function of probe-sample separation, Appl. Phys. Lett. 116, 202401 (2020).

[19] C. Gonzalez-Ballestero, T. Van der Sar, and O. Romero-Isart, Towards a quantum interface between spin waves and paramagnetic spin baths, Phys. Rev. B 105, 075410 (2022).

[20] L. Thiel, Z. Wang, A. Tschudin, D. Rohner, I. GutiérrezLezama, N. Ubrig, M. Gibertini, E. Giannini, F. Morpurgo, and P. Maletinsky, Probing magnetism in $2 \mathrm{~d}$ materials at the nanoscale with single-spin microscopy, Science 364, 973 (2019).

[21] G. Kurizki, P. Bertet, Y. Kubo, K. Mølmer, D. Petrosyan, P. Rabl, and J. Schmiedmayer, Quantum technologies with hybrid systems, Proc. Natl. Acad. Sci. USA 112, 3866 (2015).

[22] D. Lachance-Quirion, Y. Tabuchi, A. Gloppe, K. Usami, and Y. Nakamura, Hybrid quantum systems based on magnonics, Appl. Phys. Express 12, 070101 (2019).

[23] Y. Li, W. Zhang, V. Tyberkevych, W. Kwok, A. Hoffmann, and V. Novosad, Hybrid magnonics: Physics, circuits, and applications for coherent information processing, J. Appl. Phys. 128, 130902 (2020).

[24] D. D. Awschalom, C. H. R. Du, R. He, J. Heremans, A. Hoffmann, J. Hou, H. Kurebayashi, Y. Li, L. Liu, V. Novosad, J. Sklenar, S. Sullivan, D. Sun, H. Tang, V. Tyberkevych, C. Trevillian, A. W. Tsen, L. Weiss, W. Zhang, X. Zhang et al., Quantum engineering with hybrid magnonics systems and materials, IEEE Trans. Quantum Eng. 2, 1 (2021).
[25] R. Damon and J. Eshbach, Magnetostatic modes of a ferromagnet slab, J. Phys. Chem. Solids 19, 3 (1960).

[26] K. Wagner, A. Kákay, K. Schultheiss, A. Henschke, T. Sebastian, and H. Schultheiss, Magnetic domain walls as reconfigurable spin-wave nanochannels, Nat. Nanotechnol. 11, 432 (2016).

[27] N. Nagaosa and Y. Tokura, Topological properties and dynamics of magnetic skyrmions, Nat. Nanotechnol. 8, 899 (2013).

[28] V. Sluka, T. Schneider, R. Gallardo et al., Emission and propagation of $1 \mathrm{~d}$ and $2 \mathrm{~d}$ spin waves with nanoscale wavelengths in anisotropic spin textures, Nat. Nanotechnol. 14, 328 (2019).

[29] A. V. Chumak, V. I. Vasyuchka, A. A. Serga, and B. Hillebrands, Magnon spintronics, Nat. Phys. 11, 453 (2015).

[30] X. Wang, Y. Xiao, C. Liu et al., Electrical control of coherent spin rotation of a single-spin qubit, npj Quantum Inf. 6, 78 (2020).

[31] M. Fukami, D. R. Candido, D. D. Awschalom, and M. E. Flatté, Opportunities for long-range magnon-mediated entanglement of spin qubits via on- and off-resonant coupling, PRX Quantum 2, 040314 (2021).

[32] F. Casola, T. van der Sar, and A. Yacoby, Probing condensed matter physics with magnetometry based on nitrogen-vacancy centres in diamond, Nat. Rev. Mater. 3, 17088 (2018).

[33] Y. Dovzhenko, F. Casola, S. Schlotter, T. Zhou, F. Büttner, R. Walsworth, G. Beach, and A. Yacoby, Hybrid sensors based on color centers in diamond and piezoactive layers, Nat. Commun. 5, 4065 (2014).

[34] F. Matsukura, Y. Tokura, and H. Ohno, Control of magnetism by electric fields, Nat. Nanotechnol. 10, 209 (2015); U. Bauer, L. Yao, A. Tan, P. Agrawal, S. Emori, H. Tuller, S. Van Dijken, and G. S. D. Beach, Magneto-ionic control of interfacial magnetism, Nat. Mater. 14, 174 (2015).

[35] A. Laucht, J. Muhonen, F. Mohiyaddin, R. Kalra, J. Dehollain, S. Freer, F. Hudson, M. Veldhorst, R. Rahman, G. Klimeck, K. Itoh, D. Jamieson, J. McCallum, A. Dzurak, and A. Morello, Electrically controlling single-spin qubits in a continuous microwave field, Sci. Adv. 1, e1500022 (2015).

[36] N. Sun and G. Srinivasan, Voltage control of magnetism in multiferroic heterostructures and devices, SPIN 2, 1240004 (2012).

[37] R. Ramesh and N. A. Spaldin, Multiferroics: Progress and prospects in thin films., Nat. Mater. 6, 21 (2007).

[38] S. Zhang, Y. Zhao, X. Xiao, Y. Wu, S. Rizwan, L. Yang, P. Li, J. Wang, M. Zhu, H. Zhang, X. Jin, and X. Han, Giant electrical modulation of magnetization in Co40 Fe 40 $\mathrm{B} 20 / \mathrm{Pb}(\mathrm{Mg} 1 / 3 \mathrm{Nb} / 3) 0.7 \mathrm{Ti} 0.3 \mathrm{O} 3$ (011) heterostructure, Sci. Rep. 4, 3727 (2015).

[39] T. Shen, V. Ostwal, K. Camsari, and J. Appenzeller, Demonstration of a psuedo-magnetization based simultaneous write and read operation in a CoFeB/PMn-Pt heterostruture, Sci. Rep. 10, 10781 (2020).

[40] F. Dolde, H. Fedder, M. Doherty, T. Nöbauer, F. Rempp, G. Balasubramanian, T. Wolf, F. Reinhard, L. Hollenberg, F. Jelezko, and J. Wrachtrup, Room-temperature entanglement between single defect spins in diamond, Nat. Phys. 7, 459 (2011).

[41] M. Doherty, N. Manson, P. Delaney, F. Jelezko, J. Wrachtrup, and L. Hollenberg, The nitrogen-vacancy colour centre in diamond, Phys. Rep. 528, 1 (2013).

[42] C. Kittel, On the theory of ferromagnetic resonance absorption, Phys. Rev. 73, 155 (1948). 
[43] B. Kalinikos and A. Slavin, Theory of dipole-exchange spin wave spectrum for ferromagnetic films with mixed exchange boundary conditions, J. Phys. C 19, 7013 (1986).

[44] P. Li, Y. Zhao, S. Zhang, A. Chen, D. Li, J. Ma, Y. Liu, D. Pierce, J. Unguris, H. Piao, H. Zhang, M. Zhu, X. Zhang, X. Han, M. Pan, and C. Nan, Spatially resolved ferroelectric domain-switching-controlled magnetism in $\mathrm{Co} 40 \mathrm{Fe} 40 \mathrm{~B} 20 / \mathrm{Pb}(\mathrm{Mg} 1 / 3 \mathrm{Nb} 2 / 3) 0.7 \mathrm{Ti} 0.3 \mathrm{O} 3$ multiferroic heterostructure, ACS Appl. Mater. Interfaces 9, 2642 (2017).

[45] C. Degen, F. Reinhard, and P. Cappellaro, Quantum sensing, Rev. Mod. Phys 89, 035002 (2017).

[46] F. Guo, L. M. Belova, and R. D. McMichael, Spectroscopy and Imaging of Edge Modes in Permalloy Nanodisks, Phys. Rev. Lett. 110, 017601 (2013).

[47] A. Franson, N. Zhu, M. Kurfman, S. Chilcote, D. Candido, K. Buchanan, M. Flatté, H. X. Tang, and E. Johnston-Halperin, Low-damping ferromagnetic resonance in electron-beam patterned, high-q vanadium tetracyanoethylene magnon cavities, APL Mater. 7, 121113 (2019); H. Yusuf, M. Chilcote, D. Candido, S. Kurfman, D. Cormode, Y. Lu, M. Flatté, and E. Johnston-Halperin, Exploring a quantum-information-relevant magnonic material: Ultralow damping at low temperature in the organic ferrimagnet v [tcne] x, AVS Quantum Sci. 3, 026801 (2021); S. Kurfman, A. Franson, P. Shah, G. Srinivasan, M. Page, and E. Johnston-Halperin, Electric-field control of strain-driven tuning of fmr in the low-loss ferrimagnetic coordination compound v [tcne] x, Bull. Am. Phys. Soc. 65 (2020).
[48] D. Hopper, H. Shulevitz, and L. Bassett, Spin readout techniques of the nitrogen-vacancy center in diamond, Micromachines 9, 437 (2018).

[49] See Supplemental Material at http://link.aps.org/supplemental/ 10.1103/PhysRevResearch.4.L012025. First, the nanomagnet calculations directly incorporate mode ellipticity (see Supplemental Materials S5). Second, the absence of excitation continuum due to mode discretization suppresses two-magnon noise [14]. Third, the observed length scale of inhomogeneity in PMN-PT due to domains is larger than the typical nanomagnet size [44]; combined with the quasiuniform mode profile of the Kittel mode, such inhomogeneities are thus expected to be less relevant for the nanomagnet geometry.

[50] S. Cherepov, P. Khalili Amiri, J. G. Alzate, K. Wong, M. Lewis, P. Upadhyaya, J. Nath, M. Bao, A. Bur, T. Wu, G. P. Carman, A. Khitun, and K. L. Wang, Electric-field-induced spin wave generation using multiferroic magnetoelectric cells, Appl. Phys. Lett. 104, 082403 (2014). J. Cui, C.-Y. Liang, E. A. Paisley, A. Sepulveda, J. F. Ihlefeld, G. P. Carman, and C. S. Lynch, Generation of localized strain in a thin film piezoelectric to control individual magnetoelectric heterostructures, ibid. 107, 092903 (2015).

[51] A. Chen, Y. Zhao, Y. Wen, L. Pan, P. Li, and X. Zhang, Full voltage manipulation of the resistance of a magnetic tunnel junction, Sci. Adv. 5, eaay5141 (2019); A. Biswas, H. Ahmad, J. Atulasimha, and S. Bandyopadhyay, Experimental demonstration of complete $180^{\circ}$ reversal of magnetization in isolated Co nanomagnets on a PMn-Pt substrate with voltage generated strain, Nano Lett. 17, 3478 (2017). 